\title{
Why Do you Often Stop your Impulsive Behavior at the Last Moment?
}

\author{
Yongchun Wang ${ }^{1,2} \cdot$ Peng Liu $^{3} \cdot$ Zhao Yao ${ }^{1,2} \cdot$ Yonghui Wang ${ }^{4,5}$
}

Published online: 30 March 2020

(C) The Psychonomic Society, Inc. 2020

\begin{abstract}
Intentional inhibition, a critical ability of human cognitive control, is the capacity to internally withhold a to-be-performed action. In the voluntary decision-making process, the cognitive system continuously generates intentional inhibition to ensure appropriate actions. However, the temporal dynamic of intentional inhibition is unclear. This study investigated the role of temporal preparation in internally generated intentional inhibition using a novel task that involved a modified free-choice task and a foreperiod paradigm. The experiment included Go, No-Go and free-choice trials, and temporal preparation was manipulated by varying the duration and constancy of the interval between the warning stimulus and target stimulus. The results showed that a high degree of temporal preparation can strengthen the intentional decision to inhibit action on free choices. By demonstrating that intentional inhibition is enhanced with increased temporal preparation, the present study illustrates how the cognitive decision-making system controls the flexibility and strategy in human behavior.
\end{abstract}

Keywords Inhibition $\cdot$ Intentional decision $\cdot$ Temporal preparation $\cdot$ Action

\section{Introduction}

Individuals must inhibit impulsive behavior, resist temptation, delay gratification, and obey contracts in their social life. All of these capacities are based on self-control, an essential feature of human cognition, which is defined as "the overriding or inhibiting of automatic, habitual, or innate behaviors, urges, emotions, or desires that would otherwise interfere with goal directed behavior" (Muraven, Shmueli, \& Burkley, 2006).

Intentional inhibition, which is the capacity to endogenously and voluntarily inhibit or cancel an about-to-be-executed action at the last moment, is the core process of self-control (Baumeister, Vohs, \& Tice, 2007). It is perceived as a "neural

Yongchun Wang

wyongchun@xidian.edu.cn

1 School of Humanities, Xidian University, 266 Xinglong Section of Xifeng Road, Xi'an 710126, Shaanxi, China

2 Shaanxi Development Strategy Research Center of Smart Society, Xi'an 710126, China

3 School of Public Management, Northwest University, Xi'an, China

4 School of Psychology, Shaanxi Normal University, Xi'an 710062, Shaanxi, China

5 Shaanxi Provincial Key Laboratory of Behavior \& Cognitive Neuroscience, Xi'an 710062, China brake" mechanism that plays a critical role in action decisions (Filevich, Kühn, \& Haggard, 2012). This capacity to internally inhibit a prepared action guarantees that the action decision system is more flexible.

Internally generated inhibition shares some features with externally triggered inhibition, for which an action will be inhibited when a participant encounters a particular stimulus, such as a No-Go stimulus or stop signal. For example, in both forms of inhibition, action preparation is already underway when inhibition occurs (Filevich et al., 2012). However, it is worth noting that these types of inhibition have different origins and neural mechanisms. Intentional inhibition is related to the medial frontal system and is generated internally by individuals themselves, but externally triggered inhibition is related to the lateral parietal-premotor system and is caused by an external signal or instruction. Therefore, in the study of intentional inhibition, the GolNo-Go task and stop signal task that are used to study externally triggered inhibition cannot be used. Few tasks are related to intentional inhibition because obvious difficulties exist in studying this process, e.g., intentional inhibition is not preceded by an external stimulus and does not result in any behavioral output (Schel, 2015; Schel, Scheres, \& Crone, 2014). Researchers usually employ the free-choice task, in which participants are asked to prepare an action and then to decide to inhibit or execute it by themselves (Brass \& 
Haggard, 2007; Haggard, Poonian, \& Walsh, 2009; Parkinson \& Haggard, 2014; Walsh, Kühn, Brass, Wenke, \& Haggard, 2010).

Response preparation, a top-down anticipatory preactivation of response structures in the temporal dimension (Coles, Gratton, Bashore, Eriksen, \& Donchin, 1985; Coull, 2009; Müller-Gethmann, Ulrich, \& Rinkenauer, 2003; Schlaghecken \& Eimer, 2001), can speed responses to Go targets (Miller, 1982, 1983; Wang et al., 2017). Thus, reaction time (RT, simple or choice RT) is a behavioral indicator of response preparation in a forced choice task. Importantly, response preparation affects not only the execution speed of an overt response (i.e., RT) but also externally triggered motor activation and inhibition (i.e., priming effect; see Wang et al., 2017). The foreperiod paradigm is a common approach used to study response preparation. In this paradigm, response preparation is controlled by varying the length of the foreperiod (i.e., the interval between the warning stimulus (S1) and target stimulus (S2); Niemi \& Näätänen, 1981). Various accounts indicate that response preparation develops during the foreperiod (see Los, Kruijne, \& Meeter, 2014).

Individuals are faced with more free choices rather than forced action in daily life, and intentional inhibition is much closer to real-life situations than externally triggered inhibition because many of our daily activities involve stopping actions based on internally generated (i.e., intentional) stop signals, rather than explicit external stimuli telling us to stop our actions. For example, you are preparing to hand in an examination paper, when you suddenly get the feeling that you should check it again, and you hold back. Additionally, you walk into a cake shop and are just about to buy a cake, when you seem to hear a voice telling you that you need to lose weight, and you leave empty-handed. On these occasions, people tend to endogenously generate inhibition at the last moment before the execution of an action. That is, people are more likely to withhold an action when action preparation in the temporal dimension is enhanced.

It is generally considered that behavior has a "goal-planaction" serial nature (see Haruno, Wolpert, \& Kawato, 2001). Filevich et al. (2012) suggested that the inner-action loop (i.e., the goal-plan-action feedback loop) is monitored by an outerdistal loop, which contains an "impact predictor" that estimates distal consequences. If the forthcoming action is mismatched with the long-term interest, the ongoing operation of the inner-action loop may be suspended. The intentional inhibition signal is generated by the outer loop. We speculate that intentional inhibition is dynamic; specifically, the strength of inhibition is amplified rapidly with an increase in motor preparation in the temporal dimension, and the action is more likely to be cancelled. The current study attempts to verify this dynamic inhibition mechanism.

We developed a novel experimental paradigm that involved a modified free-choice task and a foreperiod paradigm to investigate whether and how temporal preparation modulates internally generated intentional inhibition. In the experiment (see Fig. 1), participants were presented with a warning stimulus (S1) followed by a target stimulus (S2) consisting of three types, a Go target (a square, participants must perform the specified action; $50 \%$ of all trials), a No-Go target (a circle, participants need not perform any action; $16 \%$ of all trials) and a free target (a triangle, participants had free choice to either act or inhibit their action; 34\% of all trials). The different proportions used in this setting were intended to make the Go stimulus sufficiently frequent, and thus the action is prepotent (see Parkinson \& Haggard, 2015). Additionally, the S1S2 inter-stimulus interval (ISI) was manipulated and consisted of two conditions: (1) a fixed ISI condition, in which the ISIs were presented in blocks, and (2) a variable ISI condition, in which the ISIs were randomized. RTs would be shortened with an increasing S1-S2 ISI for the Go target, as participants were preparing to respond as soon as the $\mathrm{S} 1$ appeared. However, "getting ready" requires time; therefore, the response preparation was greater at the longer S1-S2 ISI. Additionally, RTs for the Go target would be shorter in the fixed ISI condition than in the variable ISI condition because with fixed ISIs, participants had a chance to adjust the timing of their response preparation so that it was relatively enhanced, even for a shorter ISI; with variable ISIs, such an adjustment is not possible (see Wang et al., 2018). More importantly, according to relevant literature and our daily experience mentioned above, we speculate that greater temporal preparation results in increased intentional inhibition. Therefore, we predicted that the percentage of no action in free-choice trials would be higher for the long ISI condition than for the short ISI condition and would be higher for the fixed ISI condition than for the variable ISI condition.

\section{Experiment}

\section{Method}

\section{Participants}

Thirty college students (11 males) whose ages ranged from 17 to 24 (mean age $=21.03$ years) participated in return for monetary compensation of $¥ 20$ each. A priori power analysis using G*Power determined the appropriate sample size by estimating a moderate effect size for the interaction in a $2 \times 2 \times 2$ within-subjects analysis of variance (ANOVA). A power analysis, in which 24 participants provided .95 power by setting the alpha level at .05 , determined a medium-size effect $(f=.25$; Cohen, 1992). Based on this analysis, we recruited 30 students to participate in this experiment. All participants were right-handed and had normal or corrected-to-normal vision. Informed consent was obtained from each participant. 


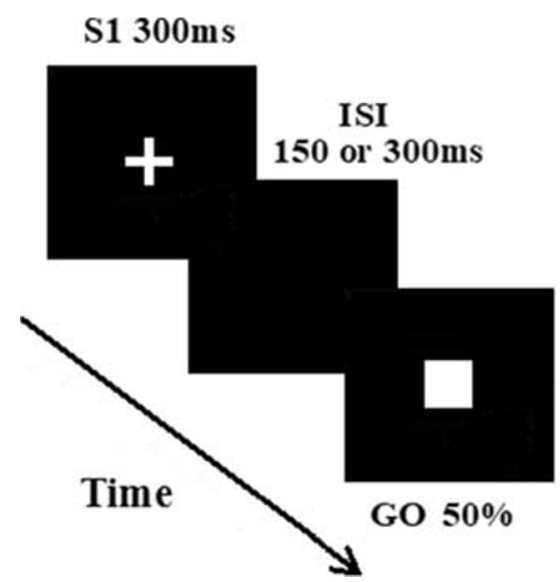

Fig. 1 Schematic representation of the trial procedure in the experiment

\section{Stimuli and Apparatus}

A cross served as the warning stimulus (S1), and a square (Go target), circle (No-Go target) and triangle (free target) served as the target stimuli (S2), which subtended a visual angle of $1^{\circ} \times 1^{\circ}$. All stimuli appeared at the fixation center and were white upon a black background. Displays were presented on a 17-in monitor. Participants viewed displays from a distance of $60 \mathrm{~cm}$ in a dimly lit room.

\section{Procedure}

The experimental procedure is shown in Fig.1. Each trial began with S1 (300 ms), which was followed after a delay of either 150 or $300 \mathrm{~ms}$ by one of the three types of the S2 (100 ms). Participants were instructed that each trial would start with the $\mathrm{S} 1$ and would be followed by a random stimulus of the three types of S2. They were instructed to prepare a keypress action from the onset of S1. Participants were further instructed that the square was a Go signal, upon which they must perform the prepared action as rapidly as possible; the circle was a No-Go signal, upon which they should withhold the keypress action; and the triangle was a free-choice signal, upon which they were asked to make an in-the-moment decision regarding whether to execute or inhibit the keypress action. In free-choice trials, participants were encouraged to not be extremely biased toward one choice or the other (see Parkinson \& Haggard, 2014; Parkinson \& Haggard, 2015). The allowed response window was from onset of any S2 to $1000 \mathrm{~ms}$.

The experiment consisted of two conditions ( 6 blocks per condition): a fixed ISI condition and a variable ISI condition. In the fixed ISI condition, the S1-S2 ISIs ( 150 or $300 \mathrm{~ms}$ ) were presented in blocks (60 trials per block), whereas in the variable ISI condition, the ISIs were randomized within each block (60 trials per block). Participants were presented a total of 360 Go trials ( $50 \%$, square shapes), 120 No-Go trials ( $16 \%$, circle shapes) and 240 free-choice trials (34\%, triangle

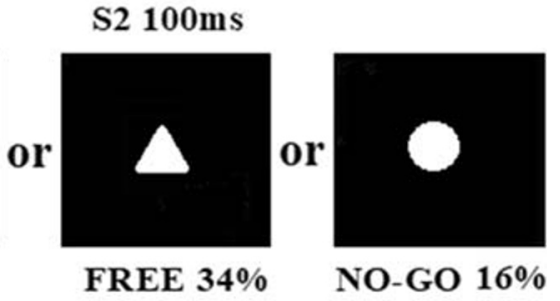

shapes). These three types of trials were randomized and presented in a fixed proportion within each block. The sequences of the ISIs in the fixed ISI condition were randomized for each participant. The sequence of the fixed ISI and variable ISI conditions was systematically balanced across participants. A practice block was presented prior to the formal experiment. Data acquisition lasted approximately $40 \mathrm{~min}$ in total.

\section{Results}

RTs were submitted to repeated-measure ANOVA with trial type (Go trial, free-choice Go trial), ISI constancy (fixed, variable) and S1-S2 ISI (150, $300 \mathrm{~ms})$ as within-subject factors. The results are shown in Fig. 2. The mean RT was shorter for the Go trial than for the free-choice Go trial (477 ms vs. $542 \mathrm{~ms} ;[F(1,29)=295.38, p<.001, M S E=847.99$, $\left.\left.\eta_{\mathrm{p}}{ }^{2}=.91\right]\right)$ and was shorter for the fixed ISI condition than for the variable ISI condition (504 ms vs. $515 \mathrm{~ms}$; $[F(1$, $\left.\left.29)=8.58, p=.007, M S E=810.11, \eta_{\mathrm{p}}{ }^{2}=.23\right]\right)$. The mean RT was longer for the 150 -ms ISI condition than for the 300-ms ISI condition (i.e., the forewarning effect, $515 \mathrm{~ms}$ vs. $503 \mathrm{~ms} ;[F(1,29)=18.93, p<.001, M S E=414.61$, $\left.\left.\eta_{\mathrm{p}}{ }^{2}=.40\right]\right)$. The two-way interaction of trial type and S1-S2 ISI was significant $[F(1,29)=8.80, p=.006, M S E=234.92$, $\eta_{\mathrm{p}}{ }^{2}=.23$ ], with the forewarning effect being weaker in the free-choice Go trial $(544 \mathrm{~ms}$ and $539 \mathrm{~ms}$; $[F(1,29)=2.37$, $p=.14])$ than in the Go trial (486 $\mathrm{ms}$ and $468 \mathrm{~ms}$; $[F(1$, $29)=34.98, p<.001])$. No other significant effect was observed $[F \mathrm{~S}<3.45, p \mathrm{~s}>.073]$.

Repeated-measure ANOVAs were performed to assess the no-response rates on free-choice trials, the miss rates on Go trials and the false alarm rates on the No-Go trials for the factors of ISI constancy (fixed, variable) and S1-S2 ISI (150, $300 \mathrm{~ms}$ ). For the no-response rates on free-choice trials, the main effect of ISI constancy was significant $[F(1,29)=5.69$, $\left.p=.024, M S E=180.91, \eta_{\mathrm{p}}{ }^{2}=.16\right]$, with a higher no-response rate for the fixed ISI than for the variable ISI condition 

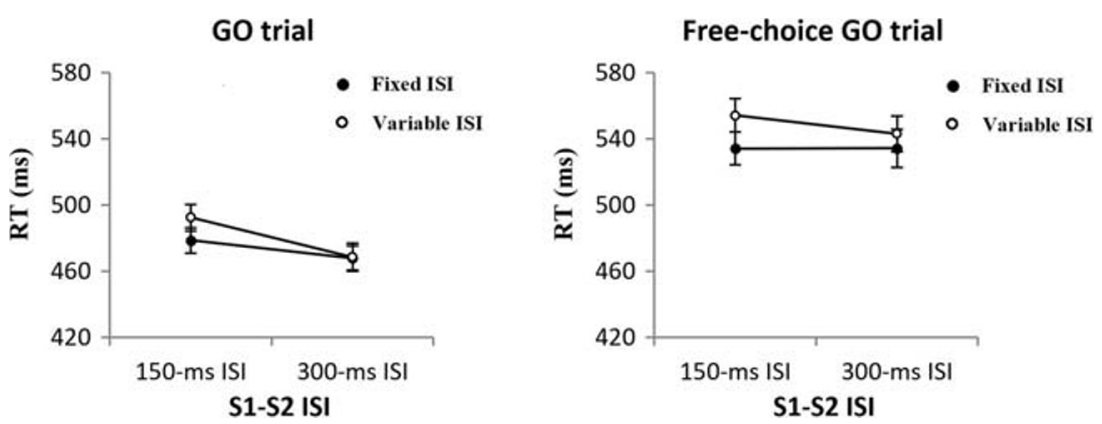

Fig. 2 Mean RTs obtained in the Go and free-choice Go trials for fixed and variable ISI conditions in the experiment. Error bars represent one standard error of the mean (SE)

(49.47\% vs. $43.61 \%)$. The main effect of S1-S2 ISI was significant $\left[F(1,29)=11.86, p=.002, M S E=119.01, \eta_{\mathrm{p}}{ }^{2}=.29\right]$, with a significant forewarning effect (i.e., the no-response rate was higher for the 300-ms ISI than for the 150-ms ISI condition $(49.97 \%$ vs. $43.11 \%)$ ). The two-way interaction of ISI constancy and ISI was significant $[F(1,29)=6.82, p=.014$, $\left.M S E=85.84, \eta_{\mathrm{p}}{ }^{2}=.19\right]$, showing that the forewarning effect was stronger in the fixed ISI condition $(43.83 \%$ and $55.11 \%$ for the 150- and 300-ms ISI, respectively; $[F(1,29)=11.26$, $p=.002])$ than in the variable ISI condition $(42.39 \%$ and $44.84 \%$ for the 150 - and 300-ms ISI, respectively; $[F(1$, 29) $=2.53, p=.12]$ ).

Regarding the miss rates on Go trials, no significant effect was observed $\left[F_{\mathrm{S}}<1.40, p \mathrm{~s}>.25\right]$. For the false alarm rates on the No-Go trials, the main effect of the S1-S2 ISI was significant $\left[F(1,29)=23.37, p<.001, M S E=23.71, \eta_{\mathrm{p}}{ }^{2}=.45\right]$, with a higher false alarm rate for the $150-\mathrm{ms}$ ISI than for the 300 -ms ISI condition $(8.41 \%$ vs. $4.11 \%)$. No other significant effect was observed $[F \mathrm{~S}<3.10, p \mathrm{~s}>.09]$. The results are shown in Fig. 3.

\section{Discussion}

This study is the first, to our knowledge, to examine the influence of temporal preparation on internally generated intentional inhibition. We used a mixed paradigm that include Go, No-Go and free-choice targets. Temporal preparation was manipulated by varying the duration and constancy of the S1-S2 ISI. The RT decreased with an increasing S1-S2 ISI, and it was shorter in the fixed ISI condition than in the variable ISI condition. These results, as we predicted, indicated that temporal preparation is greater at longer S1-S2 ISIs and with more constant ISIs. Additionally, in the Go trials, the short RT in the 300-ms ISI for variable ISI condition (nearly equals to that in the 300 -ms ISI for fixed ISI condition) probably reflects a hazard function, i.e., once $150 \mathrm{~ms}$ has passed with no stimulus, the subjects know that the stimulus must occur at $300 \mathrm{~ms}$, so the RT will be shortened. More importantly, the no-response rate on free-choice trials was relatively high with short ISIs and increased with longer ISIs in the fixed ISI condition; however, the no-response rate was low for short ISIs and did not significantly increase with long ISIs in the variable ISI condition. That is, withholding an action is more likely when participants know they can take more time before responding or not responding. This result suggests that enhanced temporal preparation can strengthen the intentional decision to withhold action on free choices.

Additionally, the RT was longer for free-choice Go trials than for Go trials, probably because there is an attempted inhibitory process in free-choice Go trials. The forewarning effect in the free-choice Go trials ( $5 \mathrm{~ms}, p=.14$ ) was not significant and was weaker than that in the Go trials $(14 \mathrm{~ms}$, $p<.001)$; that is, the RT of free-choice Go trials was not significantly decreased with the increase in ISI. This suggests

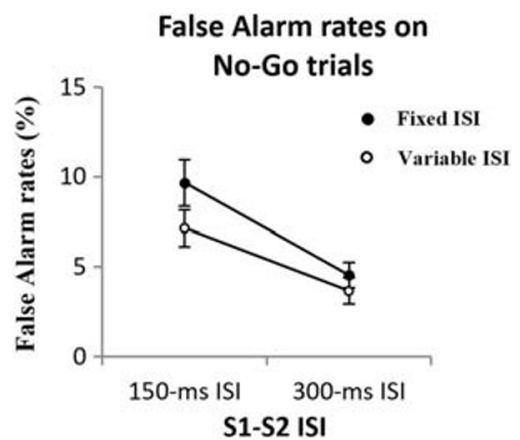

Fig. 3 The no-response rates on free-choice trials (left panel) and the false alarm rates on the No-Go trials (right panel) in the experiment. Error bars represent one SE

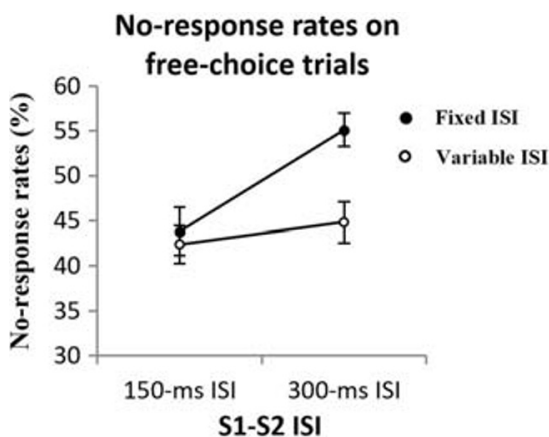


that the high temporal preparation (e.g., long ISI) may strengthen the attempted inhibitory process, a competition between action and inhibition results in the insignificant forewarning effect in the free-choice Go trials. The false alarm rate on No-Go trials was higher for short ISI, demonstrating that it is difficult for participants to adjust their impulsive action with low temporal preparation (e.g., short ISI).

The outer-distal loop iteratively checks whether the current action produced in the inner loop matches the long-term goals and continuously generates intentional inhibition (Filevich et al., 2012). The dynamic inhibition model emphasizes that the strength of intentional inhibition is not constant but is reinforced rapidly with increasing temporal action preparation within the inner loop. Therefore, when temporal preparation is greater, the action is more likely to be cancelled in the voluntary decision-making process.

The process of modulation of intentional inhibition by temporal preparation in voluntary decisions is suggested to occur as follows: (i) The S1 triggers action preparation regardless of the S1-S2 ISI. (ii-a) The action preparation increases sharply in the fixed ISI condition, is amplified substantially when the S2 is presented after a short ISI and is continually amplified with a long ISI. (ii-b) However, for variable ISIs, action preparation increases slowly, thus little amplification occurs when the target is presented after a short ISI, and amplification is not significantly increased even with a long ISI (e.g., $300 \mathrm{~ms}$ ). According to the dynamic inhibition model for voluntary decisions, greater temporal preparation results in stronger intentional inhibition. Therefore, the no response rates for freechoice trials were relatively higher when a short ISI was presented and increased when a long ISI was presented in the fixed ISI condition. However, the no-response rates were low when a short ISI was presented and did not significantly increase with a long ISI in the variable ISI condition.

Note that one may wonder when the inhibition occurs in the Go/No-Go/choose paradigm, that is, whether the decision to inhibit is made in real time during each trial or is predecided in advance. In fact, Parkinson and Haggard (2015) provided electrophysiological evidence that inhibition occurs in the moment in the Go/No-Go/choose paradigm. They found that the same N2 amplitude occurred between approximately 200 and $300 \mathrm{~ms}$ after the target onset in No-Go trials, free-choice response trials and free-choice no-response trials. That is, the inhibition in free-choice trials, like in No-Go trials, occurs in real time during each trial.

To avoid premature or impulsive behavior, our cognitive system can internally withhold a prepotent action after its initial preparation (Atas \& Cleeremans, 2015; Walsh et al., 2010). More remarkably, internally generated intentional inhibition is amplified sharply with increasing temporal preparation. This capacity allows the cognitive decision-making system to exhibit more strategic and flexible behavior.
Open Practices Statement The data and materials for all experiments are available as electronic supplementary material, and none of the experiments were preregistered.

Funding This research was supported by the MOE (Ministry of Education in China) Project of Humanities and Social Sciences to Yongchun Wang (20YJC190022) and the Fundamental Research Funds for the Central Universities to Yongchun Wang (20101196829), and also was supported by Shaanxi Development Strategy Research Center of Smart Society.

\section{Compliance with Ethical Standards}

Conflict of Interest The authors declare no competing financial interests.

\section{References}

Atas, A., \& Cleeremans, A. (2015). The temporal dynamic of automatic inhibition of irrelevant actions. Journal of Experimental Psychology: Human Perception and Performance, 41(2), 289305. https://doi.org/10.1037/a0038654

Baumeister, R. F., Vohs, K. D., \& Tice, D. M. (2007). The strength model of self-control. Current Directions in Psychological Science, 16(6), 351-355. https://doi.org/10.2307/20183234

Brass, M., \& Haggard, P. (2007). To do or not to do: the neural signature of self-control. Journal of Neuroscience, 27(34), 9141-9145. https:// doi.org/10.1523/jneurosci.0924-07.2007

Cohen, J. (1992). A power primer. Psychological Bulletin, 112(1), 155159. https://doi.org/10.1037/0033-2909.112.1.155

Coles, M. G. H., Gratton, G., Bashore, T. R., Eriksen, C. M., \& Donchin, E. (1985). A psychophysiological investigation of the continuous flow model of human information processing. Journal of Experimental Psychology: Human Perception and Performance, 11(5), 529-533. https://doi.org/10.1037/0096-1523.11.5.529

Coull, J. T. (2009). Neural substrates of mounting temporal expectation. PLOS Biology, 7(8), e1000166. https://doi.org/10.1371/journal. pbio. 1000166

Filevich, E., Simone Kühn, \& Haggard, P. (2012). Intentional inhibition in human action: The power of 'no'. Neuroscience \& Biobehavioral Reviews, 36(4), 1107-1118. https://doi.org/10.1016/j.neubiorev. 2012.01.006

Haggard, P., Poonian, S., Walsh, E. (2009). Representing the consequences of intentionally inhibited actions. Brain Research, 1286, 106-113. https://doi.org/10.1016/j.brainres.2009.06.020

Haruno, M., Wolpert, D. M., \& Kawato, M. (2001). Mosaic model for sensorimotor learning and control. Neural Computation, 13(10), 2201-2220. https://doi.org/10.1162/089976601750541778

Los, S. A., Kruijne, W., \& Meeter, M. (2014). Outlines of a multiple trace theory of temporal preparation. Frontiers in Psychology, 5(3), 1058. https://doi.org/10.3389/fpsyg.2014.01058

Miller, J. (1982). Discrete versus continuous stage models of human information processing: In search of partial output. Journal of Experimental Psychology: Human Perception and Performance, 8(2), 273-296. https://doi.org/10.1037/0096-1523.8.2.273

Miller, J. (1983). Can Response Preparation Begin Before Stimulus Recognition Finishes? Journal of Experimental Psychology: Human Perception and Performance, 9(2), 161-182. https://doi. org/10.1037/0096-1523.9.2.161

Müller-Gethmann, H., Ulrich, R., \& Rinkenauer, G. (2003). Locus of the effect of temporal preparation: Evidence from the lateralized 
readiness potential. Psychophysiology 40(4), 597-611. https://doi. org/10.1111/1469-8986.00061

Muraven, M., Shmueli, D., \& Burkley, E. (2006). Conserving self-control strength. Journal of Personality and Social Psychology, 91(3), 524 537. https://doi.org/10.1037/0022-3514.91.3.524.

Niemi, P., \& Näätänen, R. (1981). Foreperiod and simple reaction time. Psychological Bulletin, 89(1), 133-162. https://doi.org/10.1037/ 0033-2909.89.1.133

Parkinson, J., \& Haggard, P. (2014). Subliminal priming of intentional inhibition. Cognition, 130(2), 255-265. https://doi.org/10.1016/j. cognition.2013.11.005

Parkinson, J., \& Haggard, P. (2015). Choosing to stop: Responses evoked by externally triggered and internally generated inhibition identify a neural mechanism of will. Journal of Cognitive Neuroscience, 27(10), 1948-1956. https://doi.org/10.1162/jocn_a_00830

Schel, M. A. (2015). Free won't: Neurobiological bases of the development of intentional inhibition. http://hdl.handle.net/1887/32213

Schel, M. A., Scheres, A., \& Crone, E. A. (2014). New perspectives on self-control development: highlighting the role of intentional inhibition. Neuropsychologia, 65, 236-246. https://doi.org/10.1016/j. neuropsychologia.2014.08.022
Schlaghecken, F., \& Eimer, M. (2001). Partial response activation to masked primes is not dependent on response readiness. Perceptual and Motor Skills. 92(1), 208-222. https://doi.org/10.2466/pms. 2001.92.1.208

Walsh, E., Kühn, S., Brass, M., Wenke, D., Haggard, P. (2010). EEG activations during intentional inhibition of voluntary action: an electrophysiological correlate of self-control? Neuropsychologia, 48(2), 619-626. https://doi.org/10.1016/j.neuropsychologia.2009.10.026

Wang, Y., Li, Y., Liu, D., Zou, M., Zhang, B., Wang, Y. (2018). The role of response readiness in subliminal visuomotor processes. Consciousness and Cognition, 68, 23-32. https://doi.org/10.1016/ j.concog.2018.12.002

Wang, Y., Wang, Y., Liu, P., Di, M., Gong, Y., Zhao, L., \& Chen, Q. (2017). Response readiness modulates the development of association-based automaticity in masked priming. Attention Perception \& Psychophysics, 79(3), 820-832. https://doi.org/10. 3758/s13414-016-1275-4

Publisher's note Springer Nature remains neutral with regard to jurisdictional claims in published maps and institutional affiliations. 